\title{
MeCCSA PGN 2013 Conference Special Issue Introduction
}

\author{
SAM WARD, University of Nottingham \\ Journal Editor
}

This special issue of Networking Knowledge really showcases the breadth and richness of the research being done by MeCCSA's postgraduate community. Based on papers given at the PGN's annual conference at the University of East Anglia last year, the articles below cover topics ranging from the promotion of the latest Bond film to the movement of Baltic artists around Europe, and methodologies including original archival discoveries, various forms of discourse analysis, and interviews with industrial and creative professionals. This shows that, as media forms and methods of communication become evermore diverse, fragmented, converged and fast-changing in the digital age, the future of research in these fields promises a suitably multi-faceted and adaptable approach to the challenge of understanding it all. The issue stays true to this journal's title, bringing together, as did the conference, a fascinatingly interconnected set of subjects. Indeed, networking knowledge in this way is, I think, an indispensable habit for all scholars within MeCCSAs subject, if we are to remain relevant and effective as researchers in the current climate of fragmentation: working with and through the links between our many various questions, fields, methodologies and institutional priorities, and seeing them as innovative opportunities, rather than inconvenient barriers.

The issue starts with four articles that each add a different perspective on the broad theme of reviewing cinema's history. Julia Bohlmann's contribution gives insight into a previously untouched moment in the history of film censorship debates, focusing the broad issue of moral panic about early cinema's impact on children through the perspective of regional (Scottish) jurisdictions. Filipa Antunes then picks up nicely on the same topic, but in a quite different 'transitional moment', at the other end of the twentieth century. Her article considers the ambiguities surrounding a new film classification in the United States in 1980s that arguably created a new sub-genre, the 'tween' horror. Through a case study of a single film, the article opens onto a set of issues that have been hotly contested in media and film studies in recent years beyond classification itself: genre, demographics and fan discourse. Adam Scales' article on Nightmare on Elm Street 2 continues on the theme of horror, synthesising analysis of textual and reception discourses in order to understand the complex and ambiguous construction of 'alternative' - in this case queer - audiences. Moving from horror to an even more slippery label, Michael Ahmed then gives a timely consideration of what the 'exploitation film' might be in the British context. Like Scales, Ahmed shows how our frameworks for understanding exactly how films are received and defined by audiences and critics must not be rigid, but instead allow for the inevitable overlaps, fluid interconnections and confusion between categories.

The following three articles examine media paratexts. Stephanie Janes offers a detailed explanation of the promotional alternate reality game, with original interviews with some of the creators and players of these new multi-media marketing experiences. Her interrogation of the player and 
'puppetmaster' roles shows a complex negotation of power, collaboration and ownership at work that unsettles previous distinctions made between producers and users. Boundaries are questioned too in Dolores Moreno's article, which encourages the developing field of screenplay studies to consider the after-lives of film scripts - in terms of finished films, award recognition for writing, and published screenplay - as equally important a part of 'screenplay discourse' as the strictly preproduction process of conception. Again, negotiations of power and ownership sit behind Moreno's discussion, especially powerful in her critique of the pedagogical consensus on how screenwriters should be trained. Concluding this section, Sarah Kelley gives a survey of the means by which Skyfall was made into a comeback hit for the James Bond franchise. Isolating the key themes of nationality, nostalgia and notability, this article is an engaging reminder of the way in which contemporary media promotion works dynamically across platforms and cultural contexts and simultaneously towards a multitude of strategic ends.

We return to the economy of cultural capital at work in generic classification with Patrick Bingham's article on the television series Pretty Little Liars. This article also returns to the topics of teenage audiences and homosexual narratives, the two intersecting in the question of how 'drama', 'mystery' and 'teen TV' have been set into a value-laden hierarchy by the programme's promoters and critics. Emma Duester presents her detailed ethnographic study of artists based in the Baltics, arguing that a new conception of 'mobility', rather than 'migration', is needed to account for the trans-national and fluidly networked experience of her subjects. This shifts the focus to the art world and to geography, showing how the impact of globalisation on creative industries throws up complex forms of experience that resist simplistic oppositions like 'liminal' and 'central'. Finally, Thomas James Scott brings the issue back to where it started, with the early decades of featurelength cinema. Scott considers the representation of another example of mobility - that of Irish nationals to the United States - drawing on numerous instances from the archives to trace how depictions of Irish immigrants was refined and adapted as the medium matured, leading us to consider how ethnic difference, and immigration itself, were gradually built in to Hollywood's image of the American Dream.

With such an eclectic mix of topics and approaches, there really is something for all scholars in this issue. With that in mind, it serves as a perfect launch-pad for the new policy at Networking Knowledge of inviting articles on an open basis, to complement its usual themed collections. It is hoped that this will allow for the publication of more ground-breaking postgraduate and early career research even if it doesn't fit within any of the upcoming themes, and so broaden further the network's discussions and discoveries. I also hope it provides ample inspiration for new postgraduates to join the network and all members to submit their work to this year's PGN conference at the University of Leeds. It is sure to be just as dynamic and stimulating as the work represented here. 